\title{
Prognosis of COVID-19 in pregnancy: Protocol for an overview of systematic reviews
}

\section{AUTHORS:}

\section{Laura Vergara-Merino}

ORCID: 0000-0002-0004-3604

School of Medicine, Cochrane Chile Associated Centre, Universidad de Valparaíso, Chile.

\section{Javier Pérez-Bracchiglione}

ORCID:0000-0001-8738-2184

Interdisciplinary Centre for Health Studies (CIESAL). Universidad de Valparaíso. Cochrane Chile Associated Centre. Valparaíso, Chile

\section{Nicolás Meza}

ORCID: 0000-0001-9505-0358

Interdisciplinary Centre for Health Studies (CIESAL), Universidad de Valparaíso, Cochrane Chile Associated Centre, Valparaíso, Chile

\section{Constanza Couve}

ORCID: 0000-0002-8015-2332

Department of Gynecology and Obstetrics, Faculty of Medicine, Universidad de Valparaíso, Chile

\section{Cynthia Carrasco}

ORCID: 0000-0003-4746-0787

Interdisciplinary Centre for Health Studies (CIESAL). Universidad de Valparaíso. Cochrane Chile Associated Centre. Valparaíso, Chile

\section{Luis Ortiz-Muñoz}

ORCID: 0000-0001-6449-2153

UC Evidence Center, Cochrane Chile Associated Center, Pontificia Universidad Católica de Chile, Santiago, Chile

\section{Gabriel Rada}

ORCID: 0000-0003-2435-0710

Fundación Epistemonikos, Santiago, Chile

UC Evidence Center, Cochrane Chile Associated Center, Pontificia Universidad Católica de Chile, Santiago, Chile

Internal Medicine Department, Faculty of Medicine, Pontificia Universidad Católica de Chile, Santiago, Chile 


\section{COVID-19 L·OVE Working Group}

\section{Corresponding author:}

Name: Javier Pérez-Bracchiglione

Email address:

javier.perez.b@gmail.com

Postal address: Angamos 655, Viña del Mar, Chile. 


\section{ABSTRACT}

\section{Objective}

We aim to map and summarise the current evidence about COVID-19 prognosis in pregnant women.

\section{Design}

This is the protocol of an overview of systematic reviews.

\section{Data sources}

We will conduct comprehensive searches in PubMed/MEDLINE, Embase, Cochrane Central Register of Controlled Trials (CENTRAL), grey literature, and L.OVE (Living OVerview of Evidence). L.OVE is a platform that maps PICO evidence questions from Epistemonikos database. In response to the COVID-19 emergency, L.OVE was adapted to expand its COVID-19 repository evidence as a way to gather it in one place. The search will cover the period until the day before submission to a journal.

\section{Eligibility criteria for selecting studies and methods}

We adapted an already published common protocol for multiple parallel systematic reviews and overviews of systematic reviews to the specificities of this question.

We will include all systematic reviews about COVID-19 in pregnant women.

Two reviewers will independently screen each study for eligibility, extract data, and assess the risk of bias.

\section{Ethics and dissemination}

No ethics approval is considered necessary. The results of this overview will be widely disseminated via peer-reviewed publications, social networks and traditional media.

\section{Keywords}

COVID-19, severe acute respiratory syndrome coronavirus 2, Coronavirus Infections, Systematic review, Overview, Pregnant Women, Gravidity, Pregnancy 


\section{INTRODUCTION}

COVID-19 is an infection caused by the SARS-CoV-2 coronavirus [1]. It was first identified in Wuhan, China, on December 31, 2019 [2]; eight months later, more than fifteen million cases of contagion had been identified across the globe [3].

While most cases result in mild symptoms, some might progress to pneumonia, acute respiratory distress syndrome and death [4-6]. In general population severe cases of COVID-19 are about 14\% and 5\%, critical [7]. Mayor risk factors for worst prognosis are the presence of diabetes, cardiovascular and respiratory disease [8]. The case fatality rate reported across countries, settings and age groups is highly variable, but it ranges from about $0.5 \%$ to $10 \%$ [9]. In hospitalised patients it has been reported to be higher than $10 \%$ in some centers [10].

Pregnant women are a special group of concern in this outbreak. Physiological changes in immunologic, cardiovascular, and respiratory systems may increase the severity of respiratory diseases, especially during the third trimester [11-13]. Scarce data about SARS and MERS suggests that coronaviruses infections during pregnancy are associated with adverse perinatal outcomes, with high rates of maternal and perinatal mortality, cesarean section, and preterm birth $[14,15]$.

Cases of pregnant women with COVID-19 have already been reported in several primary studies and systematic reviews [16-18]. However, there are still controversies about maternal prognosis and perinatal outcomes in pregnant women with COVID-19.

Using innovative and agile processes, taking advantage of technological tools, and resorting to the collective effort of several research groups, this overview of systematic reviews aims to map and summarise the current evidence on prognosis of COVID-19 in pregnant women.

\section{METHODS}

This manuscript complies with the 'Preferred Reporting Items for Systematic reviews and MetaAnalyses' (PRISMA-P) guidelines for reporting systematic reviews and meta-analyses [19]. A protocol stating the shared objectives and methodology of multiple evidence syntheses (systematic reviews and overviews of systematic reviews) to be conducted in parallel for different questions relevant to COVID-19 was published elsewhere [20]. This protocol was adapted to the specificities of the question assessed in this overview of systematic reviews.

\section{Search strategies}

\section{Electronic searches}

Our literature search will be devised by the team maintaining the L.OVE platform (https://app.iloveevidence.com), using the following approach: 
1. Identification of terms relevant to the population and exposure components of the search strategy, using Word2vec technology [21] to the corpus of documents available in Epistemonikos Database.

2. Discussion of terms with content and methods experts to identify relevant, irrelevant and missing terms.

3. Creation of a sensitive boolean strategy encompassing all the relevant terms

4. Iterative analysis of articles missed by the boolean strategy, and refinement of the strategy accordingly.

Our main search source will be Epistemonikos database (https://www.epistemonikos.org), a comprehensive database of systematic reviews and other types of evidence [22] with more than 300.000 systematic reviews, and over 400.000 studies included in those reviews. We will supplement this with information from Pubmed, EMBASE, the WHO International Clinical Trials Registry Platform and other 30 sources relevant to COVID-19. The list of sources that have been added to Epistemonikos is continuously expanded. This list of sources regularly screened by Epistemonikos for COVID-19 is updated regularly on our website [23].

In sum, Epistemonikos Database acts as a central repository. Only articles fulfilling Epistemonikos criteria are visible by users. The remaining articles are only accessible for members of COVID-19 L.OVE Working Group.

The searches will cover from the inception date of each database until the day before submission. No study design, publication status or language restriction will be applied to the searches in Epistemonikos or the additional searches.

The following strategy will be used to search in Epistemonikos Database. We will adapt it to the syntax of other databases.

((coronavir* OR coronovirus* OR "corona virus" OR "virus corona" OR "corono virus" OR "virus corono" OR hcov* OR "covid-19" OR covid19* OR "covid 19" OR "2019-nCoV" OR cv19* OR "cv-19" OR "cv 19" OR "n-cov" OR ncov* OR "sars-cov-2" OR "sars-cov2" OR (wuhan* AND (virus OR viruses OR viral)) OR (covid* AND (virus OR viruses OR viral)) OR "sars-cov" OR "sars cov" OR "sarscoronavirus" OR "severe acute respiratory syndrome" OR "mers-cov" OR "mers cov" OR "middle east respiratory syndrome" OR "middle-east respiratory syndrome" OR "covid-19-related" OR "SARS-CoV-2-related" OR "SARS-CoV2-related" OR "2019-nCoV-related" OR "cv-19-related" OR "ncov-related") AND ((pregnan* OR gestation* OR primigravid* OR "mid-pregnancy"))

\section{Other sources}

1. We will conduct cross-citation search in Google Scholar and Microsoft Academic, using each included study as the index reference.

2. We will review the reference list of each included study.

3. We will review the reference list of other broad synthesis identified by our search. 


\section{Eligibility criteria}

\section{Types of studies}

We will include all systematic reviews assessing prognosis of COVID-19 in pregnant women, including those reviews broader in scope than this overview that present separate data for our group of interest.

We will consider as a systematic review any secondary research including only primary studies, with an explicit search strategy in at least two databases.

We will exclude primary studies, clinical practice guidelines, overviews and other evidence synthesis studies including secondary studies.

\section{Types of participants}

We will include studies assessing pregnant women.

\section{Type of exposure}

We will include studies assessing the impact of SARS-CoV 2 infection. We will exclude any information on studies evaluating other coronavirus infections.

\section{Types of outcomes}

We will not use the outcomes as an inclusion criterion during the selection process. Any article meeting all the criteria except for the outcome criterion will be preliminarily included and evaluated in full text.

We used the core outcome set COS-COVID [24], studies contained in the COMET-initiative [25] repository, the existing guidelines and reviews and the judgement of the authors of this overview as an input to select the primary and secondary outcomes, as well as to decide upon inclusion. The review team will revise this list of outcomes, in order to incorporate ongoing efforts to define Core Outcomes Sets (e.g. COVID-19 Core Outcomes [26].

Primary outcome

- All-cause mortality (Maternal and Neonatal)

\section{Secondary outcomes}

- Maternal:
- Admission to intensive care required
- Mechanical ventilation support required
- Preterm delivery $<37$ weeks
- Preterm delivery $<34$ weeks
- Preterm rupture of membranes (PROM)
- Cesarean delivery 
- Infant:
○ Stillbirth
- Neonatal admission to special care and/or intensive care unit
- Mechanical ventilation support required
- APGAR score
- Infection status of the newborn (or products of conception)

\section{Selection of studies}

The results of the literature search in Epistemonikos database will be automatically incorporated into the L.OVE platform (automated retrieval), where they will be de-duplicated by an algorithm comparing unique identifiers (database ID, DOI, trial registry ID), and citation details (i.e. author names, journal, year of publication, volume, number, pages, article title and article abstract).

In L.OVE platform two researchers will independently screen the titles and abstracts yielded by the search against the inclusion criteria. We will obtain the full reports for all titles that appear to meet the inclusion criteria or require further analysis to decide about their inclusion.

We will record the reasons for excluding reviews in any stage of the search and outline the study selection process in a PRISMA flow diagram adapted for the purpose of this project.

\section{Extraction and management of data}

Using standardised forms, two reviewers will extract data independently from each included systematic review. We will extract all data from systematic reviews and not from primary studies.

We will collect the following characteristics of included systematic reviews: publication date, search sources and strategies, number of included studies, number of relevant included studies to our overview, assessment of evidence quality of included studies, elements of the systematic review question (patients, exposure definition and assessed outcomes), source of funding of the study and the conflicts of interest disclosed by the investigators. We will also extract synthesised results from systematic reviews, either narrative or quantitative depending on how systematic reviews present their results.

\section{Assessment of overlap}

We will build a matrix of evidence using Epistemonikos to visually examine the overlap of primary studies included along the different systematic reviews. Primary studies will be presented in the columns and systematic reviews in the rows. We will calculate the corrected covered area (CCA) for quantifying this overlap [27]. We will consider overlap as low if CCA is below 5\%, moderate if CCA is between $5 \%$ and $10 \%$, high if CCA is between $10 \%$ and $15 \%$, and very high if CCA is above $15 \%$. We will consider overlap degree when discussing our results. 


\section{Quality assessment}

Two authors will independently assess the quality of each included systematic review using ROBIS [28]. We will display this assessment graphically.

We will not assess quality of evidence of the included primary studies in the systematic reviews.

\section{Data synthesis}

We will present the results of the included systematic reviews narratively. We will not conduct a quantitative synthesis.

We will present the characteristics of each included systematic review in a table and we will summarise their results by maternal and perinatal outcomes defined above. We will graphically present overlap of primary studies and risk of bias assessment.

\section{NOTES}

\section{Acknowledgements}

The members of the COVID-19 L.OVE Working Group and Epistemonikos Foundation have made it possible to build the systems and compile the information needed by this project. Epistemonikos is a collaborative effort, based on the ongoing volunteer work of over a thousand contributors since 2012.

\section{Roles and contributions}

GR conceived the common protocol for all the reviews being conducted by the COVID-19 L.OVE Working Group. LV, NM, CV, LO, JP and GR drafted the manuscript, and all other authors contributed to it. The corresponding author is the guarantor and declares that all authors meet authorship criteria and that no other authors meeting the criteria have been omitted.

The COVID-19 L·OVE Working Group was created by Epistemonikos and a number of expert teams in order to provide decision makers with the best evidence related to COVID-19. Up-to-date information about the group and its member organisations is available here:

epistemonikos.cl/working-group

\section{Competing interests}

All authors declare no financial relationships with any organisation that might have a real or perceived interest in this work. There are no other relationships or activities that could have influenced the submitted work.

\section{Funding}

This project was not commissioned by any organisation and did not receive external funding. 
Epistemonikos Foundation is providing training, support and tools at no cost for all the members of the COVID-19 L·OVE Working Group.

\section{Ethics}

As researchers will not access information that could lead to the identification of an individual participant, obtaining ethical approval was waived

\section{Data sharing}

All data related to the project will be available. Epistemonikos Foundation will grant access to data. 


\section{REFERENCES}

[1] World Health Organization. Director-General's remarks at the media briefing on 2019-nCoV on 11 February 2020. [Internet] World Health Organization; 2020 [Accessed July 20, 2020]

Available from: https://www.who.int/dg/speeches/detail/who-director-general-s-remarks-atthe-media-briefing-on-2019-ncov-on-11-february-2020 .

[2] Hui DS, I Azhar E, Madani TA, Ntoumi F, Kock R, Dar O, et al. The continuing 2019-nCoV epidemic threat of novel coronaviruses to global health - The latest 2019 novel coronavirus outbreak in Wuhan, China. Int J Infect Dis 2020;91:264-6.

[3] Dong E, Du H, Gardner L. An interactive web-based dashboard to track COVID-19 in real time. The Lancet Infectious Diseases 2020;20:533-4. https://doi.org/10.1016/s1473-3099(20)301201.

[4] Guan W-J, Ni Z-Y, Hu Y, Liang W-H, Ou C-Q, He J-X, et al. Clinical Characteristics of Coronavirus Disease 2019 in China. N Engl J Med 2020;382:1708-20.

[5] Tavakoli A, Department of Medical Virology, School of Medicine, Iran University of Medical Sciences, Tehran, Iran, Vahdat K, The Persian Gulf Tropical Medicine Research Center, The Persian Gulf Biomedical Sciences Research Institute, Bushehr University of Medical Sciences, Bushehr, Iran, Keshavarz M, The Persian Gulf Tropical Medicine Research Center, The Persian Gulf Biomedical Sciences Research Institute, Bushehr University of Medical Sciences, Bushehr, Iran. Novel Coronavirus Disease 2019 (COVID-19): An Emerging Infectious Disease in the 21st Century. Iran South Med J 2020;22:432-50.

[6] Li L-Q, Huang T, Wang Y-Q, Wang Z-P, Liang Y, Huang T-B, et al. COVID-19 patients' clinical characteristics, discharge rate, and fatality rate of meta-analysis. J Med Virol 2020;92:577-83.

[7] Wu Z, McGoogan JM. Characteristics of and Important Lessons From the Coronavirus Disease 2019 (COVID-19) Outbreak in China: Summary of a Report of 72314 Cases From the Chinese Center for Disease Control and Prevention. JAMA 2020. https://doi.org/10.1001/jama.2020.2648.

[8] Huang C, Wang Y, Li X, Ren L, Zhao J, Hu Y, et al. Clinical features of patients infected with 2019 novel coronavirus in Wuhan, China. Lancet 2020;395:497-506.

[9] Global Covid-19 Case Fatality Rates [Internet] UK: Centre for Evidence-Based Medicine [Accessed July 29, 2020]. Available from: https://www.cebm.net/covid-19/global-covid-19-case-fatality-rates/

[10] Rodriguez-Morales AJ, Cardona-Ospina JA, Gutiérrez-Ocampo E, Villamizar-Peña R, HolguinRivera Y, Escalera-Antezana JP, et al. Clinical, laboratory and imaging features of COVID-19: A systematic review and meta-analysis. Travel Med Infect Dis 2020;34:101623.

[11] Jamieson DJ, Theiler RN, Rasmussen SA. Emerging infections and pregnancy. Emerg Infect Dis 2006;12:1638-43.

[12] Lapinsky SE. Acute respiratory failure in pregnancy. Obstetric Medicine 2015;8:126-32. https://doi.org/10.1177/1753495×15589223.

[13] Schwartz DA. An Analysis of 38 Pregnant Women with COVID-19, Their Newborn Infants, and Maternal-Fetal Transmission of SARS-CoV-2: Maternal Coronavirus Infections and Pregnancy Outcomes. Arch Pathol Lab Med 2020. https://doi.org/10.5858/arpa.2020-0901-SA.

[14] Schwartz DA, Graham AL. Potential Maternal and Infant Outcomes from Coronavirus 2019nCoV (SARS-CoV-2) Infecting Pregnant Women: Lessons from SARS, MERS, and Other Human Coronavirus Infections. Viruses 2020;12:194. https://doi.org/10.3390/v12020194.

[15] Di Mascio D, Khalil A, Saccone G, Rizzo G, Buca D, Liberati M, et al. Outcome of Coronavirus spectrum infections (SARS, MERS, COVID 1 -19) during pregnancy: a systematic review and meta-analysis. Am J Obstet Gynecol MFM 2020:100107. 
[16] Mullins E, Evans D, Viner R, O'Brien P, Morris E. CORONAVIRUS IN PREGNANCY AND DELIVERY: RAPID REVIEW AND EXPERT CONSENSUS n.d. https://doi.org/10.1101/2020.03.06.20032144.

[17] Chen H, Guo J, Wang C, Luo F, Yu X, Zhang W, et al. Clinical characteristics and intrauterine vertical transmission potential of COVID-19 infection in nine pregnant women: a retrospective review of medical records. Lancet 2020;395:809-15.

[18] Yang Z, Wang M, Zhu Z, Liu Y. Coronavirus disease 2019 (COVID-19) and pregnancy: a systematic review. J Matern Fetal Neonatal Med 2020:1-4.

[19] Moher D, Shamseer L, Clarke M, Ghersi D, Liberati A, Petticrew M, et al. Preferred reporting items for systematic review and meta-analysis protocols (PRISMA-P) 2015 statement. Syst Rev 2015;4:1.

[20] Rada G, Verdugo-Paiva F, Ávila C, Morel-Marambio M, Bravo-Jeria R, Pesce F, et al. Evidence synthesis relevant to COVID-19: a protocol for multiple systematic reviews and overviews of systematic reviews. Medwave 2020;20:e7868.

[21] dperezrada. dperezrada/keywords2vec. GitHub n.d. https://github.com/dperezrada/keywords2vec (accessed July 12, 2020).

[22] Website n.d. https://www.epistemonikos.org/en/about_us/methods (accessed July 12, 2020).

[23] Living Overview of the Evidence (L·OVE) n.d. https://app.iloveevidence.com/covid-19 (accessed July 12, 2020).

[24] Jin X, Pang B, Zhang J, Liu Q, Yang Z, Feng J, et al. Core Outcome Set for Clinical Trials on Coronavirus Disease 2019 (COS-COVID). Engineering 2020. https://doi.org/10.1016/j.eng.2020.03.002.

[25] Briscoe KE, Haas DM. Developing a Core Outcome Set for Cesarean Delivery Maternal Infectious Morbidity Outcomes. Am J Perinatol 2020;37:436-52.

[26] admin. COVID-19-COS | COVID-19 Core Outcomes. COVID-19-COS n.d. https://www.covid-19cos.org/ (accessed July 15, 2020).

[27] Pieper D, Antoine S-L, Mathes T, Neugebauer EAM, Eikermann M. Systematic review finds overlapping reviews were not mentioned in every other overview. J Clin Epidemiol 2014;67:368-75.

[28] Whiting P, Savović J, Higgins JPT, Caldwell DM, Reeves BC, Shea B, et al. ROBIS: A new tool to assess risk of bias in systematic reviews was developed. J Clin Epidemiol 2016;69:225-34. 